\title{
Macular retinal and choroidal thickness in unilateral amblyopia using swept-source optical coherence tomography
}

Syunsuke Araki ${ }^{1}$, Atsushi Miki ${ }^{1,2 *}$, Katsutoshi Goto ${ }^{1}$, Tsutomu Yamashita ${ }^{1,2}$, Go Takizawa' ${ }^{1}$, Kazuko Haruishi ${ }^{1}$, Yoshiaki leki ${ }^{1}$, Junichi Kiryu ${ }^{1}$ and Kiyoshi Yaoeda ${ }^{3}$

\begin{abstract}
Background: To investigate macular retinal and choroidal thickness in amblyopic eyes compared to that in fellow and normal eyes using swept-source optical coherence tomography (SS-OCT).

Methods: This study examined 31 patients with hyperopic anisometropic amblyopia (6.9 \pm 3.8 years, mean \pm standard deviation), 15 patients with strabismic amblyopia without anisometropia (7.9 \pm 4.2 years), and 24 age-matched controls ( $7.8 \pm 3.3$ years). Retinal and choroidal thickness was measured by 3D scans using SS-OCT. A 6-mm area around the fovea was automatically analyzed using the Early Treatment Diabetic Retinopathy Study map. The thickness from SS-OCT was corrected for magnification error using individual axial length, spherical refraction, cylinder refraction, and corneal radius. Retinal thickness was divided into the macular retinal nerve fiber layer (mRNFL), ganglion cell layer + inner plexiform layer $(G C L+I P L)$, ganglion cell complex (GCC), and the inner limiting membrane to the retinal pigment epithelium (ILM-RPE) thickness. Retinal and choroidal thickness was compared among amblyopic, fellow, and normal eyes.

Results: In both amblyopia groups, there was no significant difference in the mRNFL, GCL+IPL, and GCC thicknesses among the amblyopic, fellow, and control eyes. In the anisometropic amblyopia group, choroidal thickness (subfovea, center $1 \mathrm{~mm}$, nasal and inferior of the inner ring, nasal of the outer ring, and center $6 \mathrm{~mm}$ ) of amblyopic eyes were significantly greater than that of fellow and normal eyes. In contrast, none of the choroidal thicknesses were significantly different among the investigated eyes in the strabismic amblyopia group.

Conclusions: We found no significant difference in inner retinal thickness in patients with unilateral amblyopia. Although there were significant differences in choroidal thickness with hyperopic anisometropic amblyopia, there was no significant difference for the strabismic amblyopia. The discrepancy in choroidal thickness between the two types of amblyopia may be due to both differences in ocular size and underlying mechanism.
\end{abstract}

Keywords: Amblyopia, Retinal thickness, Choroidal thickness, Optical coherence tomography

\section{Background}

Amblyopia, which is a visual disorder characterized by subnormal visual acuity (VA) and contrast sensitivity in one or both eyes, is caused by either visual deprivation or abnormal binocular interactions [1]. Many studies that investigated the pathogenesis of amblyopia,

\footnotetext{
* Correspondence: amiki@tc5.so-net.ne.jp

'Department of Ophthalmology, Kawasaki Medical School, 577 Matsushima, Kurashiki, Okayama 701-0192, Japan

${ }^{2}$ Department of Sensory Science, Faculty of Health Science and Technology, Kawasaki University of Medical Welfare, 288 Matsushima, Kurashiki, Okayama 701-0193, Japan

Full list of author information is available at the end of the article
}

including animal experiments by Hubel and Wiesel in the 1960s, have found morphological and functional abnormalities in the visual cortex and lateral geniculate nucleus [2-4]. In recent years, dysfunction in the lateral geniculate nucleus as well as in the visual cortex has also been found in human amblyopes $[5,6]$.

On the other hand, Ikeda [7] performed neurophysiological experiments in cats and reported that underdevelopment of the retinal ganglion cells was associated with amblyopia. Furthermore, an electrophysiological experiment reported finding a functional disturbance of the retina in human amblyopes [8]. However, these 
retinal abnormalities could not be confirmed during a subsequent examination by Hess [9]. Nonetheless, it has yet to be definitively established that the retina of amblyopes is absolutely normal.

The recent use of optical coherence tomography (OCT) has made it possible to quickly and non-invasively measure the retinal structure in humans. Spectral-domain OCT (SD-OCT) has especially improved the spatial resolution and scan speed, thereby enabling a detailed retinal analysis. Yen et al. [10] reported that the circumpapillary retinal nerve fiber layer (cpRNFL) of refractive amblyopic eyes was thicker than that observed in the normal fellow eyes when using time-domain OCT. The OCT findings reported by Li et al. [11] suggested that the foveal thickness in amblyopic eyes was greater than that in visually normal control eyes. However, it is still unclear as to why cpRNFL or foveal thickness in amblyopic eyes is thicker than that found in normal fellow eyes.

In addition, the use of the enhanced depth imaging (EDI) technique with SD-OCT has enabled imaging of the choroid [12]. The choroid accounts for $80-90 \%$ of the whole ocular blood flow and plays an important role in maintaining the retinal structure and function. Nishi et al. [13] used the EDI system with SD-OCT and reported that the subfoveal choroid of eyes with hyperopic anisometropic amblyopia was significantly thicker than that of the fellow eye and the age-matched controls. However, as of yet it has not been possible to use the EDI system with SD-OCT to measure the detailed choroidal thickness map using the 3D scan. In addition, another drawback of the EDI system is that it is difficult to clearly view the retina and choroid at the same time, as the retinal image quality decreases when we maximize the choroid image quality. Also, in order to be able to obtain a clear averaged image, it is necessary to acquire a large number of images. However, poor fixation during the acquisition time might preclude being able to perform successful scanning.

Recently, swept-source OCT (SS-OCT) has been used, as it can overcome the shortcomings of EDI. As SS-OCT is supposed to be able to reduce the poor images due to poor fixation, this means that a highly-detailed scan can be obtained even in the children with unstable fixation. Moreover, the use of the long central wavelength of 1,050 $\mathrm{nm}$ enables simultaneous visualization of the retina and the choroid. In addition, the use of the built-in automatic analysis software makes it possible to perform a map analysis of the choroidal thickness. However, to the best of our knowledge, there have been no reports that have used SS-OCT to investigate the retinal and choroidal thicknesses in amblyopia. Thus, the purpose of the current study was to use SS-OCT to evaluate the macular retinal and choroidal thickness in unilateral amblyopia due to anisometropia or strabismus.

\section{Methods}

This study adhered to the tenets of the Declaration of Helsinki and was approved by the Institutional Review Board committee of Kawasaki Medical School. This study was designed as an observational case series and conducted from April 2013 until June 2016 in the Department of Ophthalmology at Kawasaki Medical School Hospital. Verbal informed consent for the examinations was obtained from each patient or one of the parents of each patient.

All of the enrolled patients were diagnosed with unilateral amblyopia and underwent SS-OCT examination. Ophthalmologic examinations performed in all patients included best-corrected visual acuity (BCVA), intraocular pressure, cycloplegic refraction, axial length (AL), cover and cover-uncover test, extraocular movements, slit-lamp, and funduscopy. The refraction was measured by the Auto Ref / Kerato / Tonometer RKT-7700 (NIDEK Co., Ltd., Gamagori, Japan). The AL was measured by an IOL Master device (Carl Zeiss Meditec AG, Jena, Germany).

Unilateral amblyopia was defined as a condition where the decimal BCVAs were less than 0.8 in the amblyopic eye due to anisometropia or strabismus and more than 1.0 in the fellow eye. For the statistical analysis, the decimal BCVA was transformed into a logarithm of the minimum angle of resolution (logMAR) unit. Anisometropia was defined as an interocular difference in refraction (spherical equivalent) of more than 2.0 diopters (D). Patients with strabismic amblyopia had manifest strabismus on the cover test and a spherical equivalent interocular difference in refraction of less than $2.0 \mathrm{D}$. The presence or absence of a history of amblyopia treatment was not considered. Patients with ocular disorders, a history of intraocular surgery, systemic disease that may have had an influence on the retinal or choroidal thickness, and children who did not sufficiently cooperate for the OCT examination were excluded from the study.

This study also enrolled 24 right eyes of 24 agematched normal controls. The children in the control group had a decimal BCVA that was greater than 1.0, and did not have anisometropia, manifest strabismus, ocular disorders in either eye, or systemic disease that may have had an influence on the retinal or choroidal thickness.

\section{Measurement of the retinal and choroidal thickness}

SS-OCT was used to measure the retinal and choroidal thickness of the macula. The SS-OCT used for the measurements was the DRI OCT-1 Atlantis ${ }^{\bullet}$ (Topcon Corporation, Tokyo, Japan). The macular 3D scan (512 $\times 256$ A scans/ $0.8 \mathrm{sec}$ ) program of the built-in automatic analysis software was used for the measurements of the retinal and choroidal thicknesses, which included 
the macular retinal nerve fiber layer (mRNFL), ganglion cell layer + inner plexiform layer $(\mathrm{GCL}+\mathrm{IPL})$, ganglion cell complex (GCC), inner limiting membrane to the outer border of the retinal pigment epithelium (ILMRPE), and choroidal thickness (Fig. 1). The thickness from SS-OCT was corrected for magnification error using individual $\mathrm{AL}$, spherical refraction, cylinder refraction, and corneal radius.

The retinal and choroidal thickness was analyzed for each of the eyes in 9 regions of the macula in accordance with the Early Treatment Diabetic Retinopathy Study (ETDRS) [14]. Three concentric macular regions were defined, with radii of $0.5 \mathrm{~mm}$ (center $1 \mathrm{~mm}$ ), 0.5 to $1.5 \mathrm{~mm}$ (inner ring), and 1.5 to $3.0 \mathrm{~mm}$ (outer ring). Inner and outer rings were divided into four quadrants: superior, nasal, inferior, and temporal. Foveal minimum thickness (FMT) and subfoveal choroidal thickness (SFCT) were also analyzed (Fig. 2).

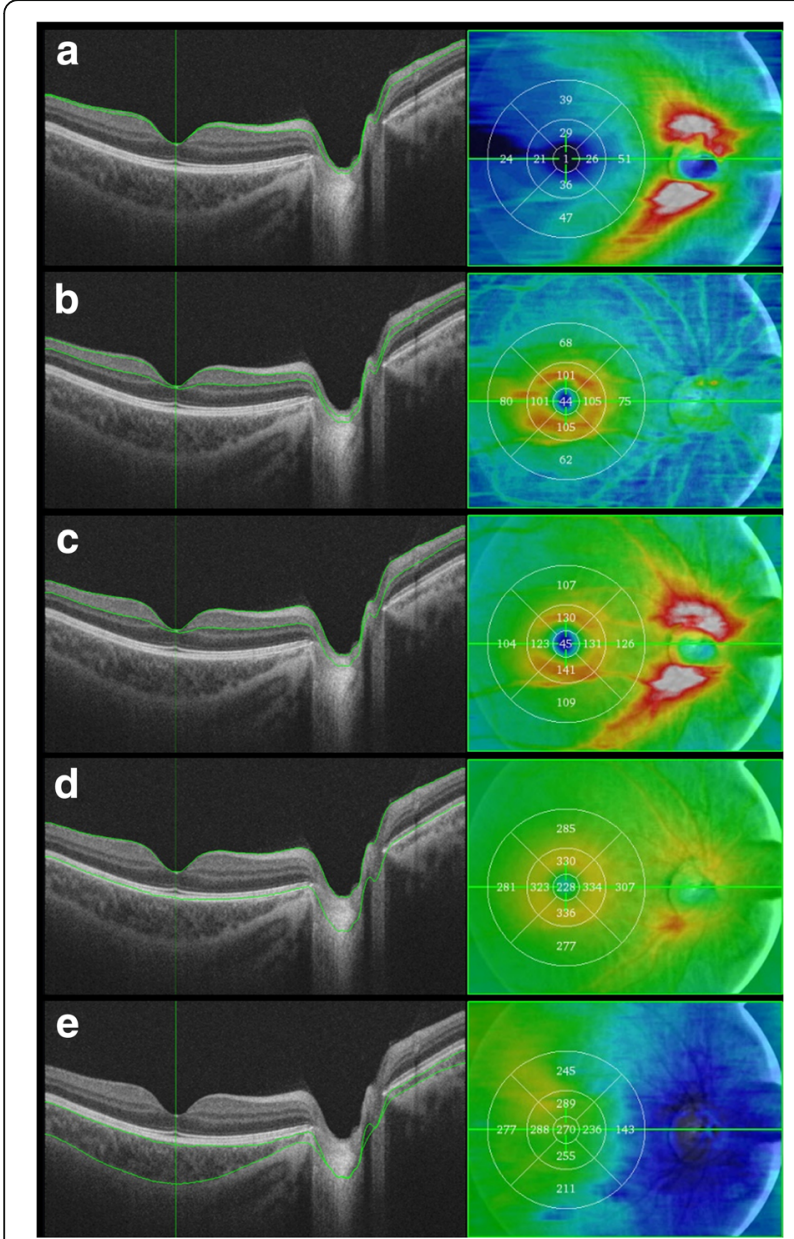

Fig. 1 Retinal and choroidal thickness determined by swept-source optical coherence tomography. The retinal and choroidal thicknesses measured included (a) macular retinal nerve fiber layer, (b) ganglion cell layer + inner plexiform layer, (c) ganglion cell complex, (d) inner limiting membrane to the retinal pigment epithelium, and (e) choroidal thickness

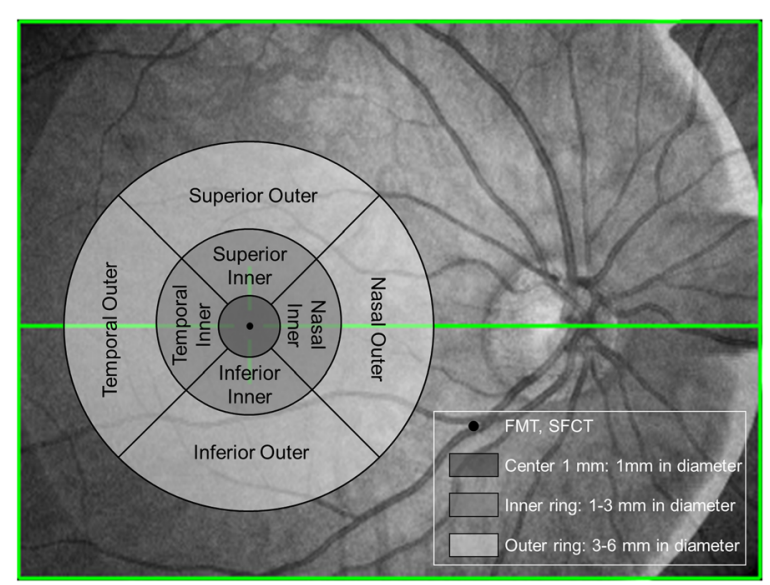

Fig. 2 Analyzed regions of retinal and choroidal thickness using the Early Treatment Diabetic Retinopathy Study map. Three concentric macular regions were defined, with radii of $0.5 \mathrm{~mm}$ (center $1 \mathrm{~mm}$ ), 0.5 to $1.5 \mathrm{~mm}$ (inner ring), and 1.5 to $3.0 \mathrm{~mm}$ (outer ring). Inner and outer rings were divided into four quadrants: superior, nasal, inferior, and temporal. Foveal minimum thickness and subfoveal choroidal thickness were also analyzed

An experienced technician (S.A.) performed all of the SS-OCT examinations after confirming the pupil diameter of the subjects was more than $4.0 \mathrm{~mm}$. All SS-OCT examinations were performed between 9:00 AM and 12:00 PM to avoid any inclusion of diurnal variations in the choroidal thickness [15]. When there was a segmentation error in the automated analysis, S.A. performed a manual modification on the measurement of the choroidal thickness. However, the data in which segmentation was difficult to obtain due to signal attenuation were excluded from the study. The segmentation error was defined to be present if at least one of two experienced technicians (S.A. and K.G.) judged that the segmentation used for the measurements of the retinal or choroidal thickness was impossible.

\section{Statistical analyses}

The statistical analysis was performed using the Bell Curve for Excel version 2.0 software program (Social Survey Research Information Co., Ltd., Tokyo, Japan). Data are presented as the means \pm standard deviations. A one-way analysis of variance (ANOVA) was used to compare the average age, and a chi-square test was used to compare the genders among each of the groups (anisometropic amblyopia, strabismic amblyopia, and normal control groups). Multiple comparisons using the Bonferroni post hoc test were performed if there was a significant difference in the one-way ANOVA. The BCVA, refraction (spherical equivalent), and AL for the amblyopic, fellow and normal control eyes were compared using the paired and 2-sample t-tests. The average retinal or choroidal thicknesses among the amblyopic, 
fellow, and normal control eyes were compared by a oneway analysis of covariance (ANCOVA), which was controlled for the AL. The correlation of the differences for the retinal or choroidal thickness in the fovea or center $6 \mathrm{~mm}$ versus the differences in the BCVA between the amblyopic and fellow eyes was determined using Pearson's correlation coefficient. The correlation between the ILMRPE thickness and the choroidal thickness in the fovea or center $6 \mathrm{~mm}$ was also determined using Pearson's correlation coefficient. The reproducibility of the judgment for the segmentation used in the measurements of the retinal or choroidal thickness was determined using kappa coefficient. For all of these analyses, $p$-values less than 0.05 were considered to be statistically significant.

\section{Results}

\section{Demographic Data}

This study enrolled 51 patients with unilateral amblyopia and 24 normal control subjects. All the subjects were Japanese. The study analyzed a total of 92 eyes of 46 patients with unilateral amblyopia and 24 right eyes of 24 normal control subjects (age: $7.8 \pm 3.3$ years). The study excluded 5 unilateral amblyopia patients due to the poor SS-OCT image quality. These five patients, in whom the segmentation of choroid from sclera was impossible, were associated with severe hyperopia. The reproducibility of the judgment for the segmentation error was excellent $(\kappa=0.88, p<0.001)$. Among the 46 patients with unilateral amblyopia, there were 31 anisometropic amblyopes (age: $6.9 \pm 3.8$ years), and 15 strabismic amblyopes (age: $7.9 \pm 4.2$ years, with 11 patients exhibiting esotropia and 4 patients exhibiting exotropia).

Table 1 shows the clinical data for all of the subjects. No significant differences were observed between the anisometropic, strabismic, and normal control groups with regard to age $(p=0.54)$ and gender $(p=0.95)$.

The $\log$ MAR in anisometropic or strabismic amblyopic eyes was significantly worse than that observed in the fellow eyes and normal control eyes $(p<0.001$ for both comparisons). The logMAR in the anisometropic amblyopic eyes was significantly worse than that observed in the strabismic amblyopic eyes $(p=0.02)$.

The refraction in the anisometropic or strabismic amblyopic eyes was more hyperopic than that found for the corresponding fellow eyes $(p<0.001$ for both comparisons), and was more hyperopic in the fellow eyes than in the normal control eyes $(p<0.001$ for both comparisons).

In the anisometropic group, the $\mathrm{AL}$ in the amblyopic eyes $(21.20 \pm 0.95 \mathrm{~mm})$ was shorter than that of the fellow eyes $(22.21 \pm 1.10 \mathrm{~mm})(p<0.001)$ and the normal control eyes $(22.74 \pm 1.06 \mathrm{~mm})(p<$ 0.001 ), with no significant difference observed between the fellow and normal control eyes $(p=0.074)$. In the strabismic group, there was also no significant difference observed in the AL between the amblyopic $(21.97 \pm 1.17 \mathrm{~mm})$ and the fellow eyes $(21.99 \pm 1.17$ $\mathrm{mm})(p=0.80)$. In addition, the AL in the normal control eyes was larger than that found for the amblyopic $(p=0.038)$ and fellow eyes $(p=0.043)$.

\section{Macular inner retinal thickness}

Tables 2, 3, 4, 5, 6 and 7 show the mean mRNFL, GCL+IPL, and GCC thicknesses in patients with unilateral amblyopia and the controls obtained when using SS-OCT.

In both amblyopia groups, there was no significant difference in the mRNFL, GCL+IPL, and GCC thicknesses among the amblyopic, fellow, and normal control eyes for all of the sectors (Tables 2, 3, 4, 5, 6 and 7).

\section{Macular ILM-RPE thickness}

Tables 8 and 9 shows the mean ILM-RPE thickness.

In the anisometropic group, the ILM-RPE thickness in the amblyopic eyes was thicker than that of the fellow eyes in the superior, nasal, and inferior sectors for the inner ring ( $p<0.05$ for all comparisons). However, there was no significant difference in the ILM-RPE thickness

Table 1 Demographic and clinical data for the amblyopia groups and the normal control group

\begin{tabular}{|c|c|c|c|c|c|}
\hline & \multicolumn{2}{|c|}{ Anisometropic Group $(n=31)$} & \multicolumn{2}{|c|}{ Strabismic Group $(n=15)$} & \multirow{2}{*}{$\begin{array}{l}\text { Normal control Group }(n=24) \\
\text { NE }\end{array}$} \\
\hline & $\overline{\mathrm{AE}}$ & $\mathrm{FE}$ & $\overline{\mathrm{AE}}$ & $\mathrm{FE}$ & \\
\hline Age & $6.9 \pm 3.8(3$ to 18$)$ & & $7.9 \pm 4.2(4$ to 21$)$ & & $7.8 \pm 3.3$ (3 to 16$)$ \\
\hline Gender (Male : Female) & $13: 18$ & & $6: 9$ & & $9: 15$ \\
\hline \multirow[t]{2}{*}{ Visual acuity (logMAR) } & $0.31 \pm 0.22$ & $-0.12 \pm 0.08$ & $0.16 \pm 0.09$ & $-0.10 \pm 0.08$ & $-0.12 \pm 0.07$ \\
\hline & (1.00 to 0.10$)$ & $(0.00$ to -0.18$)$ & (0.40 to 0.10$)$ & $(0.00$ to -0.18$)$ & (0.00 to -0.18$)$ \\
\hline \multirow[t]{2}{*}{ Refraction (diopter) } & $5.02 \pm 1.79$ & $2.07 \pm 1.79$ & $3.28 \pm 3.16$ & $2.87 \pm 3.10$ & $0.44 \pm 1.17$ \\
\hline & (1.00 to 8.00$)$ & $(-1.00$ to 5.25$)$ & $(-2.75$ to 7.00$)$ & $(-3.25$ to 6.75$)$ & $(-1.75$ to 3.25$)$ \\
\hline \multirow[t]{2}{*}{ Axial length (mm) } & $21.20 \pm 0.95$ & $22.21 \pm 1.10$ & $21.97 \pm 1.17$ & $21.99 \pm 1.17$ & $22.74 \pm 1.06$ \\
\hline & (19.61 to 23.89 ) & (20.73 to 24.91$)$ & (20.30 to 24.42 ) & (20.40 to 24.08 ) & (20.85 to 24.61$)$ \\
\hline
\end{tabular}

AE: Amblyopic eyes; FE: Fellow eyes; NE: Normal control eyes

Values are shown as mean \pm standard deviation (range) 
Table 2 mRNFL thickness in the anisometropic amblyopia and normal control eyes

\begin{tabular}{|c|c|c|c|c|c|c|}
\hline & \multicolumn{2}{|c|}{ Anisometropic amblyopia } & \multirow[b]{2}{*}{$\mathrm{NE}(n=24)$} & \multicolumn{3}{|c|}{$p$ value $^{a}$ (after adjusting for $\mathrm{AL}$ ) } \\
\hline & $\mathrm{AE}(n=31)$ & $\mathrm{FE}(n=31)$ & & AE vs FE & AE vs NE & FE vs NE \\
\hline \multicolumn{7}{|l|}{ ETDRS maps } \\
\hline Center $1 \mathrm{~mm}$ & $2.2 \pm 1.7$ & $2.5 \pm 1.8$ & $2.8 \pm 1.9$ & 0.35 & 0.81 & 0.99 \\
\hline \multicolumn{7}{|c|}{ Inner ring (1-3 mm) } \\
\hline Superior & $26.4 \pm 3.0$ & $27.0 \pm 3.3$ & $28.7 \pm 2.1$ & 0.069 & 0.71 & 0.21 \\
\hline Nasal & $20.6 \pm 3.9$ & $22.4 \pm 2.8$ & $24.3 \pm 1.7$ & 0.87 & 0.14 & 0.13 \\
\hline Inferior & $26.6 \pm 3.5$ & $28.0 \pm 4.0$ & $29.4 \pm 2.9$ & 0.18 & 0.89 & 0.92 \\
\hline Temporal & $20.2 \pm 2.8$ & $21.0 \pm 2.6$ & $22.1 \pm 1.9$ & 0.44 & 0.35 & 0.27 \\
\hline \multicolumn{7}{|c|}{ Outer ring (3-6 mm) } \\
\hline Superior & $40.6 \pm 4.8$ & $42.7 \pm 4.8$ & $42.1 \pm 3.7$ & 0.60 & 0.81 & 0.19 \\
\hline Nasal & $46.6 \pm 7.5$ & $50.0 \pm 7.7$ & $50.9 \pm 5.3$ & 0.71 & 0.90 & 0.66 \\
\hline Inferior & $41.6 \pm 4.6$ & $43.5 \pm 5.6$ & $43.3 \pm 5.4$ & 0.98 & 0.86 & 0.45 \\
\hline Temporal & $24.2 \pm 2.6$ & $24.9 \pm 2.6$ & $25.8 \pm 2.2$ & 0.62 & 0.71 & 0.33 \\
\hline Center 6 mm & $27.7 \pm 2.9$ & $29.1 \pm 3.1$ & $29.9 \pm 1.9$ & 0.59 & 0.50 & 0.96 \\
\hline
\end{tabular}

between the amblyopic and normal control eyes for all of the sectors. (Table 8)

In the strabismic group, there was no significant difference in the ILM-RPE thickness among the amblyopic, fellow, and normal control eyes for all of the sectors (Table 9).

\section{Macular choroidal thickness}

Tables 10 and 11 shows the mean choroidal thickness.

In the anisometropic group, the choroidal thickness in the amblyopic eyes was significantly thicker than that of the fellow and normal control eyes in the SFCT, center 1 $\mathrm{mm}$, nasal and inferior sectors of the inner ring, nasal sector of the outer ring, and center $6 \mathrm{~mm}(p<0.05$ for all comparisons), with no significant difference observed between the fellow and normal control eyes (Table 10).

In the strabismic group, there was no significant difference in the choroidal thickness among the amblyopic, fellow, and normal control eyes for all of the sectors (Table 11).

\section{Correlation between the difference of the BCVA and the difference of the retinal or choroidal thickness in the amblyopic and fellow eyes}

In the anisometropic group, the difference in the logMAR was not significantly correlated with the difference

Table 3 mRNFL thickness in the strabismic amblyopia and normal control eyes

\begin{tabular}{|c|c|c|c|c|c|c|}
\hline & \multicolumn{2}{|c|}{ Strabismic amblyopia } & \multirow[b]{2}{*}{$\operatorname{NE}(n=24)$} & \multicolumn{3}{|c|}{$p$ value (after adjusting for AL) } \\
\hline & $\mathrm{AE}(n=15)$ & $\mathrm{FE}(n=15)$ & & AE vs FE & AE vs NE & FE vs NE \\
\hline \multicolumn{7}{|l|}{ ETDRS maps } \\
\hline Center 1 mm & $3.5 \pm 2.7$ & $3.5 \pm 3.5$ & $2.8 \pm 1.9$ & 0.94 & 0.62 & 0.15 \\
\hline \multicolumn{7}{|c|}{ Inner ring (1-3 mm) } \\
\hline Superior & $27.9 \pm 3.7$ & $28.1 \pm 2.8$ & $28.7 \pm 2.1$ & 0.82 & 0.87 & 0.76 \\
\hline Nasal & $24.2 \pm 4.1$ & $23.9 \pm 3.1$ & $24.3 \pm 1.7$ & 0.71 & 0.28 & 0.49 \\
\hline Inferior & $28.7 \pm 3.4$ & $28.7 \pm 4.1$ & $29.4 \pm 2.9$ & 0.98 & 0.67 & 0.44 \\
\hline Temporal & $21.6 \pm 1.8$ & $20.9 \pm 3.2$ & $22.1 \pm 1.9$ & 0.62 & 0.27 & 0.73 \\
\hline \multicolumn{7}{|c|}{ Outer ring (3-6 mm) } \\
\hline Superior & $40.1 \pm 4.8$ & $39.9 \pm 2.5$ & $42.1 \pm 3.7$ & 0.87 & 0.62 & 0.78 \\
\hline Nasal & $53.1 \pm 10.4$ & $50.7 \pm 9.6$ & $50.9 \pm 5.3$ & 0.44 & 0.10 & 0.36 \\
\hline Inferior & $42.8 \pm 6.3$ & $44.3 \pm 8.6$ & $43.3 \pm 5.4$ & 0.55 & 0.45 & 0.21 \\
\hline Temporal & $24.9 \pm 1.9$ & $24.7 \pm 2.8$ & $25.8 \pm 2.2$ & 0.63 & 0.16 & 0.49 \\
\hline Center 6 mm & $29.6 \pm 3.7$ & $29.4 \pm 3.8$ & $29.9 \pm 1.9$ & 0.82 & 0.49 & 0.62 \\
\hline
\end{tabular}

AE: Amblyopic eyes; FE: Fellow eyes; NE: Normal control eyes; ETDRS: early treatment diabetic retinopathy study; AL: axial length aNCOVA; ${ }^{* *} p<0.01 ; * p<0.05$

Values are shown as mean \pm standard deviation $(\mu \mathrm{m})$ 
Table $4 \mathrm{GCL}+\mathrm{IPL}$ thickness in the anisometropic amblyopia and normal control eyes

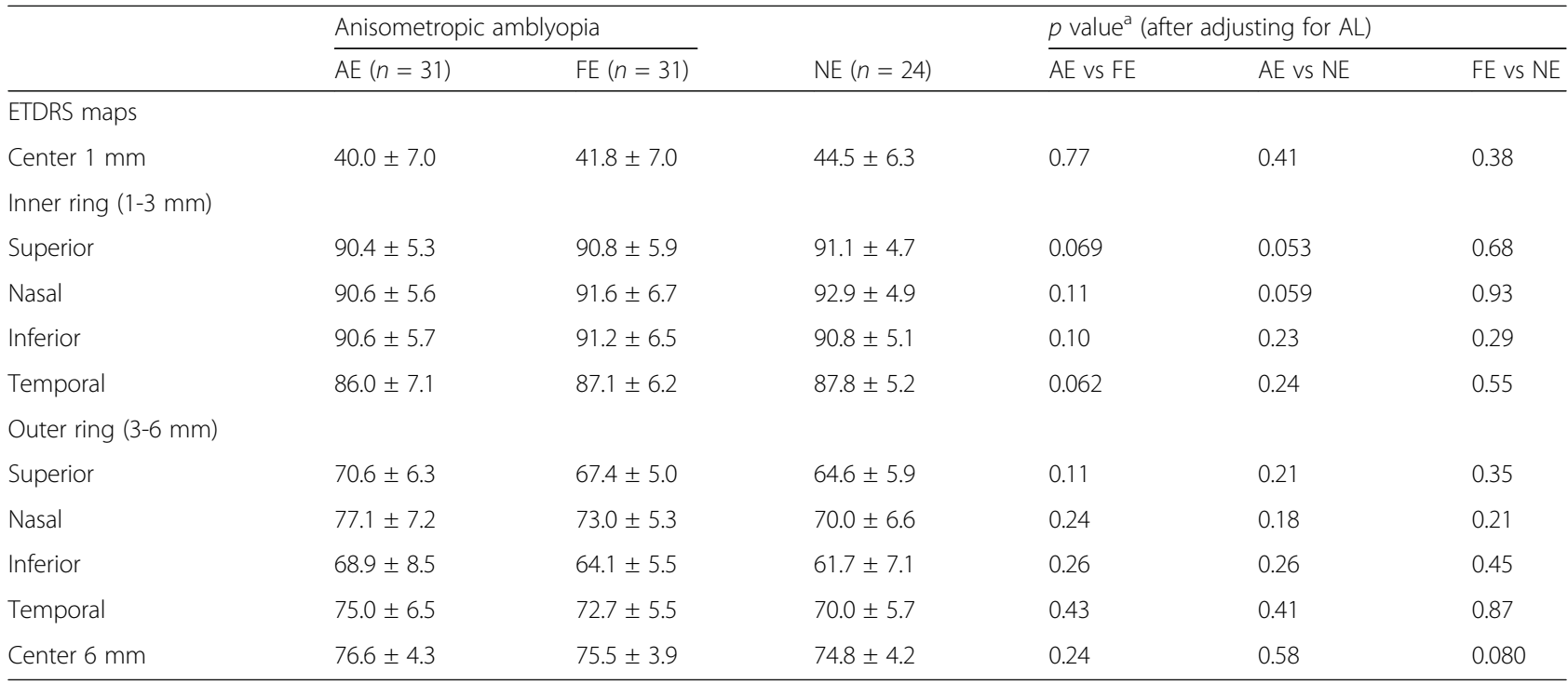

in the mRNFL thickness $(r=0.25, p=0.18), \mathrm{GCL}+\mathrm{IPL}$ thickness $(r=-0.12, p=0.52)$, GCC thickness $(r=0.037, p$ $=0.84)$, ILM-RPE thickness $(r=-0.13, p=0.47)$, FMT $(r=$ $-0.31, p=0.094)$, and SFCT $(r=0.27, p=0.15)$. Only the difference in the choroidal thickness was significantly correlated with the difference in the $\log$ MAR $(r=$ 0.37, $p=0.039$ ). In addition, there was no significant correlation between the FMT and SFCT (amblyopic eyes: $r=-0.33, p=0.069$, fellow eyes: $r=-0.19, p=$ 0.31 ) or the ILM-RPE and choroidal thickness in the center $6 \mathrm{~mm}$ (amblyopic eyes: $r=-0.23, p=0.21$, fellow eyes: $r=-0.085, p=0.65)$.
In the strabismic group, the difference in the logMAR was not significantly correlated with the difference in the mRNFL thickness $(r=0.20, p=0.47), \mathrm{GCL}+\mathrm{IPL}$ thickness $(r=-0.058, p=0.84)$, GCC thickness $(r=0.46$, $p=0.085)$, ILM-RPE thickness $(r=0.20, p=0.47)$, choroidal thickness $(r=0.048, p=0.86)$, FMT $(r=-0.076, p$ $=0.79)$, and SFCT $(r=-0.53, p=0.36)$. In addition, there was no significant correlation between the FMT and SFCT (amblyopic eyes: $r=-0.21, p=0.44$, fellow eyes: $r=-0.33, p=0.22$ ) or the ILM-RPE and choroidal thickness in the center $6 \mathrm{~mm}$ (amblyopic eyes: $r=0.22$, $p=0.43$, fellow eyes: $r=0.24, p=0.39$ ).

Table $5 \mathrm{GCL}+\mathrm{IPL}$ thickness in the strabismic amblyopia and normal control eyes

\begin{tabular}{|c|c|c|c|c|c|c|}
\hline & \multicolumn{2}{|c|}{ Strabismic amblyopia } & \multirow[b]{2}{*}{$\operatorname{NE}(n=24)$} & \multicolumn{3}{|c|}{$p$ value $^{a}$ (after adjusting for $\mathrm{AL}$ ) } \\
\hline & $\mathrm{AE}(n=15)$ & $\mathrm{FE}(n=15)$ & & AE vs FE & AE vs NE & FE vs NE \\
\hline \multicolumn{7}{|l|}{ ETDRS maps } \\
\hline Center $1 \mathrm{~mm}$ & $43.5 \pm 9.3$ & $45.1 \pm 12.1$ & $44.5 \pm 6.3$ & 0.65 & 0.63 & 0.27 \\
\hline \multicolumn{7}{|c|}{ Inner ring (1-3 mm) } \\
\hline Superior & $91.4 \pm 5.3$ & $91.6 \pm 5.2$ & $91.1 \pm 4.7$ & 0.88 & 0.93 & 0.79 \\
\hline Nasal & $93.3 \pm 7.0$ & $92.7 \pm 6.2$ & $92.9 \pm 4.9$ & 0.96 & 0.29 & 0.23 \\
\hline Inferior & $92.7 \pm 5.3$ & $90.9 \pm 5.7$ & $90.8 \pm 5.1$ & 0.32 & 0.18 & 0.29 \\
\hline Temporal & $87.0 \pm 5.6$ & $87.5 \pm 6.4$ & $87.8 \pm 5.2$ & 0.83 & 0.78 & 0.49 \\
\hline \multicolumn{7}{|c|}{ Outer ring (3-6 mm) } \\
\hline Superior & $67.7 \pm 6.4$ & $69.0 \pm 7.9$ & $64.6 \pm 5.9$ & 0.97 & 0.98 & 0.98 \\
\hline Nasal & $72.1 \pm 8.1$ & $75.5 \pm 8.1$ & $70.0 \pm 6.6$ & 0.19 & 0.88 & 0.15 \\
\hline Inferior & $63.0 \pm 6.5$ & $65.9 \pm 7.9$ & $61.7 \pm 7.1$ & 0.17 & 0.80 & 0.43 \\
\hline Temporal & $71.1 \pm 5.4$ & $73.0 \pm 5.4$ & $70.0 \pm 5.7$ & 0.83 & 0.30 & 0.21 \\
\hline Center 6 mm & $75.8 \pm 3.7$ & $76.8 \pm 3.7$ & $74.8 \pm 4.2$ & 0.94 & 0.73 & 0.67 \\
\hline
\end{tabular}

AE: Amblyopic eyes; FE: Fellow eyes; NE: Normal control eyes; ETDRS: early treatment diabetic retinopathy study; AL: axial length ${ }^{a}$ ANCOVA; ${ }^{* *} p<0.01 ;{ }^{*} p<0.05$

Values are shown as mean \pm standard deviation $(\mu \mathrm{m})$ 
Table 6 GCC thickness in the anisometropic amblyopia and normal control eyes

\begin{tabular}{|c|c|c|c|c|c|c|}
\hline & \multicolumn{2}{|c|}{ Anisometropic amblyopia } & \multirow[b]{2}{*}{$\mathrm{NE}(n=24)$} & \multicolumn{3}{|c|}{$p$ value $^{a}$ (after adjusting for $\mathrm{AL}$ ) } \\
\hline & $\mathrm{AE}(n=31)$ & $\mathrm{FE}(n=31)$ & & AE vs FE & AE vs NE & FE vs NE \\
\hline \multicolumn{7}{|l|}{ ETDRS maps } \\
\hline Center 1 mm & $42.4 \pm 7.8$ & $44.3 \pm 7.5$ & $47.3 \pm 7.6$ & 0.62 & 0.56 & 0.46 \\
\hline \multicolumn{7}{|c|}{ Inner ring (1-3 mm) } \\
\hline Superior & $116.7 \pm 7.4$ & $117.7 \pm 8.1$ & $119.8 \pm 5.7$ & 0.33 & 0.74 & 0.89 \\
\hline Nasal & $111.8 \pm 8.6$ & $113.7 \pm 8.8$ & $117.1 \pm 5.8$ & 0.071 & 0.84 & 0.42 \\
\hline Inferior & $117.2 \pm 8.1$ & $119.2 \pm 9.8$ & $120.2 \pm 6.5$ & 0.076 & 0.52 & 0.46 \\
\hline Temporal & $105.7 \pm 7.8$ & $108.3 \pm 7.6$ & $110.0 \pm 5.9$ & 0.16 & 0.83 & 0.85 \\
\hline \multicolumn{7}{|c|}{ Outer ring (3-6 mm) } \\
\hline Superior & $110.8 \pm 7.8$ & $110.2 \pm 7.6$ & $106.6 \pm 8.0$ & 0.73 & 0.68 & 0.24 \\
\hline Nasal & $123.9 \pm 7.6$ & $122.9 \pm 7.9$ & $121.0 \pm 9.0$ & 0.17 & 0.43 & 0.11 \\
\hline Inferior & $110.5 \pm 8.7$ & $107.6 \pm 7.3$ & $104.9 \pm 10.5$ & 0.36 & 0.83 & 0.59 \\
\hline Temporal & $99.2 \pm 7.6$ & $97.7 \pm 6.6$ & $95.8 \pm 6.6$ & 0.18 & 0.33 & 0.80 \\
\hline Center 6 mm & $104.2 \pm 5.8$ & $104.6 \pm 6.2$ & $104.7 \pm 5.3$ & 0.11 & 0.38 & 0.48 \\
\hline
\end{tabular}

\section{Discussion}

This study using SS-OCT measurements demonstrated that inner retinal thickness was not found to be significantly altered in unilateral amblyopia and that the choroidal thickness of amblyopic eyes in unilateral amblyopia exhibited different distinctive characteristics depending on the type of amblyopia. Specifically, in anisometropic amblyopia, the choroidal thickness in the amblyopic eyes was significantly thicker than that of the fellow and normal control eyes. In contrast, in strabismic amblyopia, there was no significant difference in the retinal or choroidal thicknesses among the amblyopic, fellow, and normal control eyes.

A large number of studies have recently used SD-OCT to assess the thickness of the retina [11] or choroid [13, 16-22] in unilateral amblyopia. However, none of the previous reports have used SS-OCT to analyze the retinal and choroidal thickness at the same time. Furthermore, these previous studies manually performed the choroidal thickness measurements only at specific points and not over defined areas [13, 16-22]. Thus, the current study is the first to use SS-OCT to evaluate the

Table 7 GCC thickness in the strabismic amblyopia and normal control eyes

\begin{tabular}{|c|c|c|c|c|c|c|}
\hline & \multicolumn{2}{|c|}{ Strabismic amblyopia } & \multirow[b]{2}{*}{$\operatorname{NE}(n=24)$} & \multicolumn{3}{|c|}{$p$ value $^{\text {a }}$ (after adjusting for $\mathrm{AL}$ ) } \\
\hline & $\mathrm{AE}(n=15)$ & $\mathrm{FE}(n=15)$ & & AE vs FE & AE vs NE & FE vs NE \\
\hline \multicolumn{7}{|l|}{ ETDRS maps } \\
\hline Center $1 \mathrm{~mm}$ & $47.2 \pm 11.5$ & $48.7 \pm 14.8$ & $47.3 \pm 7.6$ & 0.73 & 0.37 & 0.18 \\
\hline \multicolumn{7}{|c|}{ Inner ring (1-3 mm) } \\
\hline Superior & $119.3 \pm 6.8$ & $119.7 \pm 6.8$ & $119.8 \pm 5.7$ & 0.90 & 0.65 & 0.23 \\
\hline Nasal & $118.2 \pm 8.5$ & $117.1 \pm 8.6$ & $117.1 \pm 5.8$ & 0.65 & 0.26 & 0.47 \\
\hline Inferior & $121.3 \pm 7.7$ & $119.3 \pm 8.7$ & $120.2 \pm 6.5$ & 0.42 & 0.18 & 0.57 \\
\hline Temporal & $108.7 \pm 6.5$ & $108.4 \pm 7.4$ & $110.0 \pm 5.9$ & 0.89 & 0.86 & 0.92 \\
\hline \multicolumn{7}{|c|}{ Outer ring (3-6 mm) } \\
\hline Superior & $107.7 \pm 7.2$ & $107.7 \pm 5.5$ & $106.6 \pm 8.0$ & 0.52 & 0.87 & 0.99 \\
\hline Nasal & $125.1 \pm 8.3$ & $125.0 \pm 7.3$ & $121.0 \pm 9.0$ & 0.85 & 0.69 & 0.54 \\
\hline Inferior & $105.7 \pm 7.4$ & $110.1 \pm 8.2$ & $104.9 \pm 10.5$ & 0.90 & 0.83 & 0.75 \\
\hline Temporal & $96.3 \pm 5.3$ & $97.8 \pm 5.6$ & $95.8 \pm 6.6$ & 0.61 & 0.57 & 0.31 \\
\hline Center 6 mm & $105.5 \pm 5.3$ & $106.0 \pm 5.6$ & $104.7 \pm 5.3$ & 0.81 & 0.43 & 0.36 \\
\hline
\end{tabular}

AE: Amblyopic eyes; FE: Fellow eyes; NE: Normal control eyes; ETDRS: early treatment diabetic retinopathy study; AL: axial length

${ }^{a}$ ANCOVA; ${ }^{* *} p<0.01 ;{ }^{*} p<0.05$

Values are shown as mean \pm standard deviation $(\mu \mathrm{m})$ 
Table 8 ILM-RPE thickness in the anisometropic amblyopia and normal control eyes

\begin{tabular}{|c|c|c|c|c|c|c|}
\hline & Anisometrop & & & $p$ value $^{a}$ & isting for $\mathrm{Al}$ & \\
\hline & $\mathrm{AE}(n=31)$ & $\mathrm{FE}(n=31)$ & $\mathrm{NE}(n=24)$ & AE vs FE & AE vs NE & FE vs NE \\
\hline FMT & $181.6 \pm 13.1$ & $180.4 \pm 13.0$ & $186.1 \pm 15.1$ & 0.17 & 0.98 & 0.35 \\
\hline ETDRS maps & & & & & & \\
\hline Center 1 mm & $218.9 \pm 16.3$ & $219.6 \pm 14.8$ & $226.2 \pm 18.8$ & 0.21 & 0.62 & 0.47 \\
\hline Inner ring (1-3 & & & & & & \\
\hline Superior & $310.6 \pm 13.1$ & $306.5 \pm 12.3$ & $309.0 \pm 12.3$ & $0.020^{*}$ & 0.094 & 0.073 \\
\hline Nasal & $308.8 \pm 14.9$ & $305.9 \pm 13.9$ & $309.4 \pm 14.1$ & $0.047^{*}$ & 0.083 & 0.061 \\
\hline Inferior & $307.1 \pm 13.9$ & $303.6 \pm 13.6$ & $304.7 \pm 10.9$ & $0.032^{*}$ & 0.46 & 0.82 \\
\hline Temporal & $297.2 \pm 14.3$ & $295.0 \pm 12.1$ & $297.1 \pm 12.3$ & 0.059 & 0.21 & 0.15 \\
\hline Outer ring (3-6 & & & & & & \\
\hline Superior & $285.7 \pm 13.4$ & $278.2 \pm 12.6$ & $274.9 \pm 14.8$ & 0.065 & 0.59 & 0.22 \\
\hline Nasal & $300.3 \pm 13.2$ & $291.2 \pm 11.9$ & $288.6 \pm 16.1$ & 0.32 & 0.35 & 0.10 \\
\hline Inferior & $275.5 \pm 14.7$ & $265.6 \pm 12.4$ & $263.0 \pm 15.5$ & 0.13 & 0.45 & 0.85 \\
\hline Temporal & $271.9 \pm 13.3$ & $263.2 \pm 13.2$ & $261.9 \pm 12.2$ & 0.16 & 0.32 & 0.73 \\
\hline Center 6 mm & $286.6 \pm 11.4$ & $279.3 \pm 10.4$ & $278.1 \pm 12.2$ & 0.22 & 0.37 & 0.16 \\
\hline
\end{tabular}

averaged regional retinal and choroidal thickness in unilateral amblyopia using a 3D map.

In addition, previous studies that used OCT to examine the retinal or choroidal thicknesses of normal eyes have reported that the retinal thickness was influenced by age or sex, while the choroidal thickness was influenced by age, sex, AL, or refraction [23-26]. However, our current study found no significant difference in terms of sex or age between the anisometropic amblyopia, strabismic amblyopia, and normal control groups. Although the AL was significantly shorter in the anisometropic amblyopic eyes versus the fellow or normal control eyes, we compared the retinal or choroidal thickness using a statistical technique that was controlled for the AL.

\section{Macular inner retinal thickness}

Previous studies that used SD-OCT to evaluate the inner retinal thickness reported finding no significant differences in the GCL+IPL or GCC thicknesses between the amblyopic eyes and the fellow and normal control eyes [16, 27-29]. In

Table 9 ILM-RPE thickness in the strabismic amblyopia and normal control eyes

\begin{tabular}{|c|c|c|c|c|c|c|}
\hline & \multicolumn{2}{|c|}{ Strabismic amblyopia } & \multirow[b]{2}{*}{ NE $(n=24)$} & \multicolumn{3}{|c|}{$p$ value (after adjusting for $\mathrm{AL}$ ) } \\
\hline & $\mathrm{AE}(n=15)$ & $\mathrm{FE}(n=15)$ & & AE vs FE & AE vs NE & FE vs NE \\
\hline FMT & $187.7 \pm 20.2$ & $193.5 \pm 24.6$ & $186.1 \pm 15.1$ & 0.35 & 0.17 & 0.062 \\
\hline \multicolumn{7}{|l|}{ ETDRS maps } \\
\hline Center $1 \mathrm{~mm}$ & $228.5 \pm 22.4$ & $229.0 \pm 25.3$ & $226.2 \pm 18.8$ & 0.97 & 0.30 & 0.26 \\
\hline \multicolumn{7}{|c|}{ Inner ring (1-3 mm) } \\
\hline Superior & $313.1 \pm 12.9$ & $312.7 \pm 13.7$ & $309.0 \pm 12.3$ & 0.97 & 0.89 & 0.86 \\
\hline Nasal & $315.1 \pm 13.5$ & $312.9 \pm 13.2$ & $309.4 \pm 14.1$ & 0.72 & 0.36 & 0.19 \\
\hline Inferior & $311.0 \pm 11.9$ & $307.7 \pm 11.9$ & $304.7 \pm 10.9$ & 0.46 & 0.95 & 0.88 \\
\hline Temporal & $300.0 \pm 11.6$ & $298.1 \pm 12.9$ & $297.1 \pm 12.3$ & 0.97 & 0.90 & 0.88 \\
\hline \multicolumn{7}{|c|}{ Outer ring (3-6 mm) } \\
\hline Superior & $281.4 \pm 12.9$ & $279.9 \pm 13.3$ & $274.9 \pm 14.8$ & 0.73 & 0.57 & 0.90 \\
\hline Nasal & $298.7 \pm 13.8$ & $300.4 \pm 13.0$ & $288.6 \pm 16.1$ & 0.69 & 0.28 & 0.13 \\
\hline Inferior & $267.8 \pm 12.9$ & $274.4 \pm 14.6$ & $263.0 \pm 15.5$ & 0.11 & 0.95 & 0.17 \\
\hline Temporal & $266.3 \pm 12.0$ & $267.8 \pm 12.9$ & $261.9 \pm 12.2$ & 0.59 & 0.68 & 0.51 \\
\hline Center 6 mm & $284.1 \pm 10.8$ & $285.3 \pm 11.0$ & $278.1 \pm 12.2$ & 0.73 & 0.45 & 0.30 \\
\hline
\end{tabular}

AE: Amblyopic eyes; FE: Fellow eyes; NE: Normal control eyes; FMT: foveal minimum thickness; ETDRS: early treatment diabetic retinopathy study; AL: axial length ${ }^{\text {a }}$ ANCOVA; ${ }^{* *} p<0.01 ;{ }^{*} p<0.05$

Values are shown as mean \pm standard deviation $(\mu \mathrm{m})$ 
Table 10 Choroidal thickness in the anisometropic amblyopia and normal control eyes

\begin{tabular}{|c|c|c|c|c|c|c|}
\hline & \multicolumn{2}{|c|}{ Anisometropic amblyopia } & \multirow[b]{2}{*}{$\mathrm{NE}(n=24)$} & \multicolumn{3}{|c|}{$p$ value $^{\mathrm{a}}$ (after adjusting for $\mathrm{AL}$ ) } \\
\hline & $\mathrm{AE}(n=31)$ & $\mathrm{FE}(n=31)$ & & AE vs FE & AE vs NE & FE vs NE \\
\hline SFCT & $320.8 \pm 55.3$ & $274.0 \pm 55.2$ & $274.7 \pm 52.8$ & $0.016^{*}$ & $0.017^{*}$ & 0.85 \\
\hline \multicolumn{7}{|l|}{ ETDRS maps } \\
\hline Center 1 mm & $320.2 \pm 53.1$ & $274.0 \pm 53.3$ & $277.4 \pm 51.6$ & $0.011^{*}$ & $0.044^{*}$ & 0.33 \\
\hline \multicolumn{7}{|c|}{ Inner ring (1-3 mm) } \\
\hline Superior & $306.2 \pm 44.2$ & $275.5 \pm 52.7$ & $279.6 \pm 50.1$ & $0.005^{* *}$ & 0.080 & 0.31 \\
\hline Nasal & $301.7 \pm 53.5$ & $244.7 \pm 60.4$ & $247.7 \pm 51.5$ & $0.013^{*}$ & $0.007^{* *}$ & 0.33 \\
\hline Inferior & $317.4 \pm 51.4$ & $265.3 \pm 55.7$ & $275.3 \pm 45.3$ & $0.005^{* *}$ & $0.039^{*}$ & 0.18 \\
\hline Temporal & $319.6 \pm 51.4$ & $286.8 \pm 46.3$ & $293.1 \pm 54.8$ & $0.037^{*}$ & 0.21 & 0.28 \\
\hline \multicolumn{7}{|c|}{ Outer ring (3-6 mm) } \\
\hline Superior & $293.6 \pm 36.4$ & $261.6 \pm 45.0$ & $265.0 \pm 48.8$ & 0.056 & 0.396 & 0.37 \\
\hline Nasal & $251.1 \pm 51.1$ & $187.0 \pm 59.9$ & $185.7 \pm 46.7$ & $0.045^{*}$ & $0.003^{* *}$ & 0.49 \\
\hline Inferior & $287.1 \pm 49.1$ & $249.1 \pm 46.3$ & $260.4 \pm 42.9$ & $0.022^{*}$ & 0.41 & 0.16 \\
\hline Temporal & $299.8 \pm 49.3$ & $278.7 \pm 42.0$ & $286.6 \pm 54.2$ & 0.21 & 0.84 & 0.37 \\
\hline Center 6 mm & $290.2 \pm 41.6$ & $250.3 \pm 44.5$ & $255.7 \pm 44.9$ & $0.015^{*}$ & $0.049^{*}$ & 0.26 \\
\hline
\end{tabular}

contrast, Park et al. [30] reported that the GCL+IPL thickness was thinner in amblyopic eyes versus the fellow eyes. Tugcu et al. [31] further reported that the GCC thickness in anisometropic amblyopia was thicker in amblyopic eyes versus controls, while in strabismic amblyopia, it was thinner in the amblyopic eyes versus the controls. In the current study, there were no significant differences in the mRNFL, GCL + IPL, and GCC thickness found among the amblyopic, fellow, and normal control eyes.
Yen et al. [10] reported that while the RNFL thickness might be affected by refractive amblyopia, it was not affected by strabismic amblyopia. Yen et al. [10] hypothesized that amblyopia might affect the postnatal maturation of the retina, including the postnatal reduction of the optic nerve axons [32, 33], which could then lead to a measurable increase in the RNFL thickness in amblyopic eyes. However, it has also been reported that the decrease of the optic nerve axons in humans stabilizes

Table 11 Choroidal thickness in the strabismic amblyopia and normal control eyes

\begin{tabular}{|c|c|c|c|c|c|c|}
\hline & \multicolumn{2}{|c|}{ Strabismic amblyopia } & \multirow[b]{2}{*}{$\operatorname{NE}(n=24)$} & \multicolumn{3}{|c|}{ 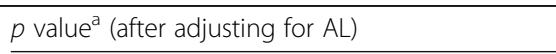 } \\
\hline & $\mathrm{AE}(n=15)$ & $\mathrm{FE}(n=15)$ & & AE vs FE & AE vs NE & FE vs NE \\
\hline SFCT & $293.1 \pm 74.3$ & $274.1 \pm 75.8$ & $274.7 \pm 52.8$ & 0.78 & 0.94 & 0.78 \\
\hline \multicolumn{7}{|l|}{ ETDRS maps } \\
\hline Center 1 mm & $293.3 \pm 68.1$ & $279.0 \pm 65.8$ & $277.4 \pm 51.6$ & 0.99 & 0.92 & 0.90 \\
\hline \multicolumn{7}{|c|}{ Inner ring (1-3 mm) } \\
\hline Superior & $268.1 \pm 40.3$ & $287.6 \pm 53.0$ & $279.6 \pm 50.1$ & 0.22 & 0.70 & 0.73 \\
\hline Nasal & $272.9 \pm 60.8$ & $265.0 \pm 66.1$ & $247.7 \pm 51.5$ & 0.73 & 0.76 & 0.85 \\
\hline Inferior & $291.6 \pm 67.5$ & $280.6 \pm 62.3$ & $275.3 \pm 45.3$ & 0.72 & 0.87 & 0.91 \\
\hline Temporal & $297.7 \pm 57.2$ & $292.3 \pm 61.5$ & $293.1 \pm 54.8$ & 0.98 & 0.96 & 0.93 \\
\hline \multicolumn{7}{|c|}{ Outer ring (3-6 mm) } \\
\hline Superior & $270.3 \pm 39.8$ & $278.6 \pm 49.1$ & $265.0 \pm 48.8$ & 0.53 & 0.49 & 0.92 \\
\hline Nasal & $220.3 \pm 56.5$ & $215.3 \pm 60.2$ & $185.7 \pm 46.7$ & 0.80 & 0.22 & 0.41 \\
\hline Inferior & $272.8 \pm 55.9$ & $267.0 \pm 61.3$ & $260.4 \pm 42.9$ & 0.79 & 0.94 & 0.77 \\
\hline Temporal & $287.9 \pm 51.8$ & $291.7 \pm 59.5$ & $286.6 \pm 54.2$ & 0.86 & 0.86 & 0.72 \\
\hline Center 6 mm & $268.1 \pm 44.3$ & $267.6 \pm 50.8$ & $255.7 \pm 44.9$ & 0.996 & 0.59 & 0.96 \\
\hline
\end{tabular}

AE: Amblyopic eyes; FE: Fellow eyes; NE: Normal control eyes; SFCT: subfoveal choroidal thickness; ETDRS: early treatment diabetic retinopathy study; AL: axial length

${ }^{\text {a ANCOVA; }}{ }^{* *} p<0.01 ;{ }^{*} p<0.05$

Values are shown as mean \pm standard deviation $(\mu \mathrm{m})$ 
around week 29 of gestation [32]. In addition, Potts et al. [33] reported that the number of retinal ganglion cells in newborn rats decreased to the same number as the number of retinal ganglion cells in adult rats on the 10th postnatal day. Therefore, neither anisometropic nor strabismic amblyopia, which exhibit a much later onset after birth, will likely prevent this normal development.

\section{Macular ILM-RPE thickness}

There have been many previous studies that have examined the macular ILM-RPE thickness of unilateral amblyopia [11, 27-31, 34-39]. Li et al. [11] performed a meta-analysis and reported that the FMT and the thickness of the center $1 \mathrm{~mm}$ and $6 \mathrm{~mm}$ in the amblyopic eyes were significantly greater than that observed in the fellow eyes $(4.6 \mu \mathrm{m}, 3.2 \mu \mathrm{m}$, and $3.5 \mu \mathrm{m}$, respectively). Additionally, only the FMT was significantly increased in the amblyopic eyes as compared with the normal control eyes.

In the anisometropic group, our current study showed that the ILM-RPE thickness in the amblyopic eyes was thicker than that of the fellow eyes only in the superior, nasal, and inferior sectors for the inner ring. However, there was no significant difference in the ILM-RPE thickness between the amblyopic and normal control eyes for all of the sectors. In addition, there was no significant difference between the fellow and normal control eyes. Thus, there is no clear explanation for our results of the ILM-RPE thickness in anisometropic amblyopia. In the strabismic group, there was also no significant difference in the ILM-RPE thickness among the amblyopic, fellow, and normal control eyes in all of the sectors.

Al-Haddad et al. [34] found that the central macular thickness was significantly increased in amblyopic eyes as compared to that of the fellow eyes in anisometropic amblyopia, but not in strabismic amblyopia. Park et al. [30] additionally reported finding no statistically significant differences in the total macular thickness between the amblyopic and normal fellow eyes in unilateral amblyopia, with these eyes also showing no significant differences in the refractive errors. Based on these findings, we believe that it is possible that a change in the ILM-RPE thickness of amblyopic eyes relates to refraction. However, AlHaddad et al. [34] also reported that anisometropia alone did not lead to such a difference, which suggests that there could possibly be a correlation between amblyopia and the development of the retinal layers.

With regard to the FMT, Huynh et al. [35] reported that amblyopic eyes had a slightly greater foveal minimum thickness than the fellow and normal control eyes in unilateral amblyopia, which was due to the anisometropia and strabismus. In addition, they found that the inner macular ring was thinner in the amblyopic versus the normal fellow eyes. As a result, these authors proposed a hypothesis that the arrest of normal postnatal changes would most likely affect the normal maturation of the macula, including the movement of Henle's fibers away from the foveola, along with a decrease in the foveal cone diameter. However, there are many other reports that have found that the FMT of amblyopic eyes was no different from the fellow and normal control eyes [20,37, 38]. The findings of our current study do not support Huynh et al.'s hypothesis [35], as we found that there were no changes in the FMT of amblyopic eyes.

Huynh et al. [35] additionally reported finding that the foveal thickening in the amblyopic eyes was more remarkable in the no treatment versus the treatment group. Pang et al. [36] also examined the FMT of amblyopic eyes and reported that it became thinner after treatment as compared to before treatment, although there was no correlation found between the FMT and VA improvement. The disagreement between our current results and the previous studies $[11,35,36]$ might be due to the fact that our present study did not exclude patients who had a history of amblyopia treatment. Additional studies that investigate classified amblyopes according to refractive error, past history of amblyopia treatment, and the depth of amblyopia will need to be undertaken in order to clarify these effects in the future.

Using the ETDRS map, Wu et al. [39] found that the macular thickness in the center $1 \mathrm{~mm}$, inner ring, or outer ring was not significantly different between the amblyopic and fellow eyes in hyperopic anisometropic amblyopia. Kim et al. [28] additionally reported that while the macular thickness (inner temporal, outer superior, outer nasal, and outer inferior) in amblyopic eyes was significantly greater than that found in the fellow eyes, these thicknesses were not significantly different between the amblyopic and normal control eyes. Our current study also found that there were no significant differences in the ILM-RPE thickness between the amblyopic and normal control eyes for all of the sectors, similar to that reported in previous studies [28, 39].

In addition, we found that there were no significant differences in the ILM-RPE thickness between the amblyopic and normal control eyes, and between the fellow and normal control eyes, although our study did reveal that the ILM-RPE thickness exhibited a significant difference between the amblyopic and fellow eyes in only a few regions. Thus, while the difference of the ILM-RPE thickness in a few regions of the inner ring between the amblyopic and fellow eyes was statistically significant, the clinical significance appears to be trivial. 


\section{Macular choroidal thickness}

In a preliminary study, Nishi et al. [13] reported that the subfoveal choroid of eyes with hyperopic anisometropic amblyopia was significantly thicker than that of the fellow eye and the age-matched controls. Many other investigators have reported that the SFCT in amblyopic eyes was greater than that of the fellow eyes with hypermetropic anisometropic amblyopia [17-22]. However, there are only a few studies that have examined the choroidal thickness in accordance with the amblyopic cause $[17,18,22]$. Similar to that for retinal thickness, whether "amblyopia influences" $[13,17]$ or "refraction influences" [40] are associated with the choroid thickening in amblyopic eyes remains controversial.

In the anisometropic group, our current study showed that the choroidal thickness in the amblyopic eyes was significantly thicker than that of the fellow and normal control eyes in the SFCT, center $1 \mathrm{~mm}$, nasal and inferior sectors of the inner ring, nasal sector of the outer ring, and the center $6 \mathrm{~mm}$. There was no significant difference between the fellow and normal control eyes. In the strabismic group, there was also no significant difference in the choroidal thickness among the amblyopic, fellow, and normal control eyes for any of the sectors.

Nishi et al. [13] described the possibility that the increased SFCT of amblyopic eyes is under the influence of the amblyopia, as the profile of the choroidal thickness in the amblyopic eyes was different from that of the fellow eyes and control eyes. In amblyopic eyes, the choroid was the thickest in the subfoveal area followed by the temporal sector, with the thinnest area found in the nasal sector. The authors hypothesized that the ocular compensation and choroidal accommodation [41] for the hyperopic defocus was suppressed in amblyopic eyes, which resulted in an increased SFCT. Since all the anisometropic amblyopia eyes in our current study had hyperopic defocus, our results do not contradict their hypothesis [13].

On the other hand, $\mathrm{Xu}$ et al. [17] suggested the possibility that FMT thickening is associated with the SFCT thickening of amblyopic eyes. Thus, a thicker retina would likely require a greater blood supply. If so, then the choroid might thicken in order to be able to supply more blood to the outer retina. However, the article by $\mathrm{Xu}$ et al. [17] did not report the retinal thicknesses found in their investigation. Although the FMT of the anisometropic amblyopia group did not exhibit a significant difference between the amblyopic and fellow eyes, the SFCT was thicker in the amblyopic eyes in our study. Moreover, we investigated the correlation between the FMT and SFCT, or ILM-RPE and choroidal thickness in the center $6 \mathrm{~mm}$ in the anisometropic amblyopia group, but found no significant correlations. Therefore, we do not believe that the thickening of the choroid occurs directly following the retinal thickening.

In the strabismic amblyopia group, our study showed that there was no significant difference in the choroidal thickness among the amblyopic, fellow, and normal control eyes. This was a novel finding as compared to previous studies that have reported that the choroid of amblyopic eyes was thick in strabismic amblyopia [17, 18, 22]. With regard to this discrepancy between the previous reports and our current study, it is possible that refraction differences between the amblyopic and fellow eyes could have influenced the results. However, similar to the strabismic amblyopia group in the current study, the findings of the previous reports $[17,18,22]$ were not complicated by the presence of anisometropia. Alternatively, there is a possibility that differences in the measurement procedures used to determine the choroidal thickness (analysis of the distance between two points set manually versus mapping analysis) could have had an effect on the choroidal thickness obtained. To the best of our knowledge, there have been no previous studies that have used SS-OCT to map the choroidal thickness. The choroidal thickness analysis in the previous studies used data that were measured as the distance from one point to another. However, these types of measurements could be easily affected by minute changes of the point actually measured. In contrast, as our present study used a map analysis, this should have resulted in more precise measurements, thereby generating more reliable data for the choroidal thickness.

In this study, changes of the choroidal thickness in amblyopic eyes were the most remarkable in the nasal region. This result was similar to the past reports $[13,17,22]$. However, it remains unclear as to why the only place that the choroidal thickening was not seen was in the temporal region in anisometropic amblyopia.

\section{Correlation between difference of the BCVA and the difference of the retinal or choroidal thickness in the amblyopic and fellow eyes}

Various studies have investigated the correlation of the retinal thickness and VA in eyes with amblyopia and reported that the VA was not correlated with the retinal thickness regardless of amblyopia type [29, 36, 42]. Our current results, which demonstrated that retinal thicknesses measured by OCT were unrelated to the degree of amblyopia, appear to support the findings of previously published reports in the literature [29, 36, 42].

Pang et al. [36], who reported finding that there was no correlation between FMT and VA improvement, 
speculated that the reason for these results was that the critically important factor that is required in high-level acuity is the foveal cone density and not the foveal thickness. In contrast, Nishi et al. [38] reported that optical treatments resulted in an improvement of the VA and also a lengthening of the outer segment in anisohypermetropic amblyopic eyes. In addition, they also found a significant correlation between the increased outer segment length and better BCVA. Based on these findings, it is possible that there are minute structural changes that occur and cannot be detected by simply measuring the retinal and choroidal thicknesses that occur in amblyopic eyes.

On the other hand, a few studies reported no correlation between the VA and choroidal thickness [17, 40]. However, in our current study, as there was a weak correlation between the difference for the choroidal thickness and the difference for the BCVA between the amblyopic and fellow eyes, the choroidal thickening in anisometropic amblyopia in the center $6 \mathrm{~mm}$ found may be related to the change in the visual function that is observed in amblyopia. A further study is necessary whether our results were due to visual function or secondary to refraction differences between the eyes.

\section{Limitation}

As a limitation for the current study, we were not able to match the refraction of the normal control eyes to that of the amblyopic eyes. If this had been possible, we might have been able to clearly determine whether the retinal or choroidal thickening was caused by hyperopia or by the amblyopia itself. However, finding a group of such controls is difficult, as eyes with high hyperopia should produce amblyopia to some extent during the time that they are uncorrected for the refractive error after birth.

\section{Conclusion}

We found no significant difference in inner retinal thickness of the patients with unilateral amblyopia. Although there were significant interocular differences for the macular choroidal thicknesses in the hyperopic anisometropic amblyopia patients, there was no significant interocular difference observed in strabismic amblyopia. The results of this study do not support the hypothesis that the change was simply due to the differences in refractive error, as we used a statistical analysis that takes the AL into consideration. Thus, the noted differences may be due to a combination of the difference in the refraction and that of the pathogenesis of the disease between the two types of amblyopia.

\section{Abbreviations}

AE: Amblyopic eyes; AL: Axial length; ANOVA: A one-way analysis of variance: BCVA: Best-corrected visual acuity; CPRNFL: Circumpapillary retinal nerve fiber layer; EDI: Enhanced depth imaging; ETDRS: Early treatment diabetic retinopathy study; FE: Fellow eyes; FMT: Foveal minimum thickness; GCC: Ganglion cell complex; GCL+IPL: Ganglion cell layer + inner plexiform layer; ILM-RPE: Inner limiting membrane to the retinal pigment epithelium; log MAR: Logarithm of the minimum angle of resolution; mRNFL: Macular retinal nerve fiber layer; NE: Normal control eyes; SD-OCT: Spectral-domain optical coherence tomography; SFCT: Subfoveal choroidal thickness; SS-OCT: Swept-source optical coherence tomography

\section{Acknowledgements}

Not applicable.

Funding

There was no funding support.

Availability of data and materials

All data are available upon request.

\section{Authors' contributions}

SA and AM designed study, collected and interpreted the data, drafted the manuscript and reviewed the literature. TY, KG, GT, and KH participated in the collection and interpretation of data. KY performed statistical analysis. YI and JK interpreted the data, and critically reviewed the manuscript. All authors read and approved the final manuscript.

\section{Ethics approval and consent to participate}

This study adhered to the tenets of the Declaration of Helsinki and was approved by the Institutional Review Board committee of Kawasaki Medical School (registration no: 2458). Additionally, verbal informed consent for the examinations was obtained from each patient or one of the parents of each patient.

\section{Consent for publication}

Not applicable.

\section{Competing interests}

The authors declare that they have no competing interests.

\section{Publisher's Note}

Springer Nature remains neutral with regard to jurisdictional claims in published maps and institutional affiliations.

\section{Author details}

${ }^{1}$ Department of Ophthalmology, Kawasaki Medical School, 577 Matsushima, Kurashiki, Okayama 701-0192, Japan. ${ }^{2}$ Department of Sensory Science, Faculty of Health Science and Technology, Kawasaki University of Medical Welfare, 288 Matsushima, Kurashiki, Okayama 701-0193, Japan. ${ }^{3}$ Yaoeda Eye Clinic, 2-1649-1 Naga-Chou, Nagaoka, Niigata 940-0053, Japan.

Received: 16 May 2017 Accepted: 29 August 2017

Published online: 15 September 2017

\section{References}

1. von Noorden GK. Amblyopia: a multidisciplinary approach. Proctor lecture. Invest Ophthalmol Vis Sci. 1985;26:1704-16.

2. Wiesel TN, Hubel DH. Single-cell responses in striate cortex of kittens deprived of vision in one eye. J Neurophysiol. 1963;26:1003-17.

3. Hubel DH, Wiesel TN. Binocular interaction in striate cortex of kittens reared with artificial squint. J Neurophysiol. 1965;28:1041-59.

4. von Noorden GK. Histological studies of the visual system in monkeys with experimental amblyopia. Invest Ophthalmol. 1973;12:727-38.

5. Miki A, Liu GT, Goldsmith ZG, Liu CS, Haselgrove JC. Decreased activation of the lateral geniculate nucleus in a patient with anisometropic amblyopia demonstrated by functional magnetic resonance imaging. Ophthalmologica. 2003;217:365-9.

6. Hess RF, Thompson B, Gole G, Mullen KT. Deficient responses from the lateral geniculate nucleus in humans with amblyopia. Eur J Neurosci. 2009;29:1064-70. 
7. Ikeda H. Visual acuity, its development and amblyopia. J R Soc Med. 1980;73:546-55.

8. Arden GB. Vaegan, Hogg CR, Powell DJ, Carter RM. Pattern ERGs are abnormal in many amblyopes. Trans Ophthalmol Soc U K. 1980;100:453-60.

9. Hess RF. Amblyopia: site unseen. Clin Exp Optom. 2001;84:321-36.

10. Yen MY, Cheng CY, Wang AG. Retinal nerve fiber layer thickness in unilateral amblyopia. Invest Ophthalmol Vis Sci. 2004;45:2224-30.

11. Li J, Ji P, Meta-analysis YM. of retinal changes in unilateral amblyopia using optical coherence tomography. Eur J Ophthalmol. 2015;25:400-9.

12. Spaide RF, Koizumi H, Pozzoni MC. Enhanced depth imaging spectral-domain optical coherence tomography. Am J Ophthalmol. 2008;146:496-500.

13. Nishi T, Ueda T, Hasegawa T, Miyata K, Ogata N. Choroidal thickness in children with hyperopic anisometropic amblyopia. Br J Ophthalmol. 2014;98:228-32.

14. Early Treatment Diabetic Retinopathy Study design and baseline patient characteristics. ETDRS report number 7. Ophthalmology. 1991;98:741-756.

15. Tan CS, Ouyang Y, Ruiz H, Sadda SR. Diurnal variation of choroidal thickness in normal, healthy subjects measured by spectral domain optical coherence tomography. Invest Ophthalmol Vis Sci. 2012;53:261-6.

16. Celik E, Çakır B, Turkoglu EB, Doğan E, Alagoz G. Evaluation of the retinal ganglion cell and choroidal thickness in young Turkish adults with hyperopic anisometropic amblyopia. Int Ophthalmol. 2016:36:515-20.

17. Xu J, Zheng J, Yu S, Sun Z, Zheng W, Qu P, et al. Macular choroidal thickness in unilateral amblyopic children. Invest Ophthalmol Vis Sci. 2014;55:7361-8.

18. Kara O, Altintas O, Karaman S, Emre E, Caglar Y. Analysis of choroidal thickness using spectral-domain OCT in children with unilateral amblyopia. J Pediatr Ophthalmol Strabismus. 2015;52:159-66.

19. Kantarci FA, Tatar MG, Uslu H, Colak HN, Yildirim A, Goker H, et al. Choroidal and peripapillary retinal nerve fiber layer thickness in adults with anisometropic amblyopia. Eur J Ophthalmol. 2015;25:437-42.

20. Mori T, Sugano Y, Maruko I, Sekiryu T. Subfoveal choroidal thickness and axial length in preschool children with hyperopic anisometropic amblyopia. Curr Eye Res. 2015;40:954-61.

21. Öner V, Bulut A. Büyüktarakçı ş, Kaim M. Influence of hyperopia and amblyopia on choroidal thickness in children. Eur J Ophthalmol. 2016; https://doi.org/10.5301/ejo.5000703

22. Aygit ED, Yilmaz I, Ozkaya A, Alkin Z, Gokyigit B, Yazici AT, et al. Choroidal thickness of children's eyes with anisometropic and strabismic amblyopia. J AAPOS. 2015;19:237-41.

23. Ooto S, Hangai M, Yoshimura N. Effects of sex and age on the normal retinal and choroidal structures on optical coherence tomography. Curr Eye Res. 2015;40:213-25.

24. Wang J, Gao X, Huang W, Wang W, Chen S, Du S, et al. Swept-source optical coherence tomography imaging of macular retinal and choroidal structures in healthy eyes. BMC Ophthalmol. 2015; https://doi.org/10.1186/ s12886-015-0110-3.

25. Nagasawa T, Mitamura Y, Katome T, Shinomiya K, Naito T, Nagasato D, et al. Macular choroidal thickness and volume in healthy pediatric individuals measured by swept-source optical coherence tomography. Invest Ophthalmol Vis Sci. 2013:54:7068-74.

26. Jin $\mathrm{P}$, Zou H, Zhu J, Xu X, Jin J, Chang TC, et al. Choroidal and retinal thickness in children with different refractive status measured by sweptsource optical coherence tomography. Am J Ophthalmol. 2016;168:164-76.

27. Firat PG, Ozsoy E, Demirel S, Cumurcu T, Gunduz A. Evaluation of peripapillary retinal nerve fiber layer, macula and ganglion cell thickness in amblyopia using spectral optical coherence tomography. Int J Ophthalmol. 2013;6:90-4.

28. Kim YW, Kim SJ, Spectral-domain YYS. optical coherence tomography analysis in deprivational amblyopia: a pilot study with unilateral pediatric cataract patients. Graefes Arch Clin Exp Ophthalmol. 2013;251:2811-9.

29. Araki S, Miki A, Yamashita T, Goto K, Haruishi K, leki Y, et al. A comparison between amblyopic and fellow eyes in unilateral amblyopia using spectral-domain optical coherence tomography. Clinical Ophthalmology. 2014;7:2199-207.

30. Park KA, Park DY, Oh Sy.Analysis of spectral-domain optical coherence tomography measurements in amblyopia: a pilot studyBr J Ophthalmol. 2011;95:1700-1706

31. Tugcu B, Araz-Ersan B, Kilic M, Erdogan ET, Yigit U, Karamursel S. The morpho-functional evaluation of retina in amblyopia. Curr Eye Res. 2013:38:802-9.

32. Provis JM, van Driel D, Billson FA, Russell P. Human fetal optic nerve: overproduction and elimination of retinal axons during development. J Comp Neurol. 1985;238:92-100
33. Potts RA, Dreher B, Bennett MR. The loss of ganglion cells in the developing retina of the rat. Brain Res. 1982;255:481-6.

34. Al-Haddad CE, Mollayess GM, Cherfan CG, Jaafar DF, Bashshur ZF. Retinal nerve fibre layer and macular thickness in amblyopia as measured by spectral-domain optical coherence tomography. Br J Ophthalmol. 2011;95: 1696-9.

35. Huynh SC, Samarawickrama C, Wang XY, Rochtchina E, Wong TY, Gole GA, et al. Macular and nerve fiber layer thickness in amblyopia: the Sydney Childhood Eye Study. Ophthalmology. 2009;116:1604-9.

36. Pang Y, Frantz KA, Block S, Goodfellow GW, Allison C. Effect of Amblyopia Treatment on Macular Thickness in Eyes With Myopic Anisometropic Amblyopia. Invest Ophthalmol Vis Sci. 2015;56:2677-83.

37. Yoon SW, Park WH, Baek SH, Kong SM. Thicknesses of macular retinal layer and peripapillary retinal nerve fiber layer in patients with hyperopic anisometropic amblyopia. Korean J Ophthalmol. 2005;19:62-7.

38. Nishi T, Ueda T, Hasegawa T, Miyata K, Ogata N. Retinal thickness in children with anisohypermetropic amblyopia. Br J Ophthalmol. 2015;99:1060-4.

39. Wu SQ, Zhu LW, Xu QB, Xu JL, Zhang Y. Macular and peripapillary retinal nerve fiber layer thickness in children with hyperopic anisometropic amblyopia. Int J Ophthalmol. 2013;6:85-9.

40. Öner V, Bulut A. Does the treatment of amblyopia normalise subfoveal choroidal thickness in amblyopic children? Clin Exp Optom. 2016; https:// doi.org/10.1111/cxo.12483

41. Wallman J, Winawer J. Homeostasis of Eye Growth and the Question of Myopia. Neuron. 2004;43:447-68.

42. Chen W, Chen J, Zhang F, Zhu X, Visual LF, outcome in isoametropic amblyopic children with high hyperopia and the effect of therapy on retinal thickness. Am J Ophthalmol. 2013;155:536-43.

\section{Submit your next manuscript to BioMed Central and we will help you at every step:}

- We accept pre-submission inquiries

- Our selector tool helps you to find the most relevant journal

- We provide round the clock customer support

- Convenient online submission

- Thorough peer review

- Inclusion in PubMed and all major indexing services

- Maximum visibility for your research

Submit your manuscript at www.biomedcentral.com/submit
) Biomed Central 